\section{Drones can monitor beach litter $40 x$ faster than people}

Marine litter is predominately plastic, which typically floats in water, causing much debris to wash up on the world's beaches. While some studies have focused on estimating the total debris floating in the ocean, estimates of beached litter are typically only compiled at the local level. On beaches, marine litter is typically estimated visually -- often with a small group of trained volunteers who sample the debris within random transects. This process can take a few hours for each beach, thus scaling up to a considerable effort if one is intending to monitor long stretches of beach multiple times a year.

Unmanned aerial vehicles (UAVs), colloquially known as drones, could offer a much faster and efficient monitoring process. The authors present a methodology for using drones to take pictures of beaches, and then using machine learning techniques to automatically count and categorize litter in these photos. Ideally, this methodology would take just one trained individual a few minutes to sample an entire beach.

The authors compared their new methodology with a traditional, manual sampling along a 350 meter-long sandy beach along the northern Red Sea. A DJI Phantom 3 Advanced quadcopter was used with a 12 megapixel camera mounted on a gimbal to steady the image. This drone is fairly cheap as far as drones go, costing less than US $\$ 1,000$ at the time of writing. It has integrated GPS and can fly for more than 20 minutes on a single charge. Testing found that the drone should be ideally flown at 10 meters above the beach with the camera mounted at a $90^{\circ}$ angle to the ground. With this setup, the drone could find debris as small as 0.5 to $0.7 \mathrm{~cm}$ long, and each track along the shore would cover 19.5 meters of beach. The drone was flown at $2 \mathrm{~m} / \mathrm{s}$, capturing an image every 2 seconds. This allowed each image to overlap with the previous image by about 10 meters, helping to properly stitch sequential images together.

Debris from was sorted into 10 categories, based on materials found from the field sampling, which included items like drink bottles, bottle caps, plastic bags, and others. A visual screening of the drone images detected about $62 \%$ of the debris that was found in the field sampling: 123 items found manually versus 76 on the images over a $56 \mathrm{~m}^{2}$ transect. However, if small items under $4 \mathrm{~cm}$ were excluded, both methodologies produced statistically similar results. The authors developed a beta version of a machine learning algorithm to automatically classify debris from the drone's imagery into the 3 most abundant categories out of the 10 . The algorithm consistently missed detecting actual debris, but also overestimated debris by classifying natural materials as litter. However, it correctly classified the debris in the 3 main categories considered that proportion of the 3 classes were statistically consistent between methodologies.

While the initial beta algorithm developed by the authors did not provide similar accuracy to manual sampling, the drone can sample the same area nearly 40 times as fast. Higher resolution imagery could help improve the automated accuracy. Future advances in "deep learning" algorithms could also help to more accurately assign debris to the proper categories.
This is a summary of: Use of Unmanned Aerial Vehicles for efficient beach litter monitoring

Accessible at: https://marxiv.org/zgtwn

Authors: Cecilia Martin, Stephen Parkes, Qiannan Zhang, Xiangliang Zhang, Matthew McCabe, Carlos Duarte

Added to MarXiv:

May 2018

Published: Marine Pollution Bulletin, 2018

\section{Suggested Citation:}

Drones can monitor beach litter 40x faster than people. OCTO (2018). DOI: 10.31230/osf.io/3g7jh

See more MarXiv summaries at https://www.marxivinfo.org/ summaries

Join the MarXiv Summaries monthly newsletter at https:// oct.to/marxivsum

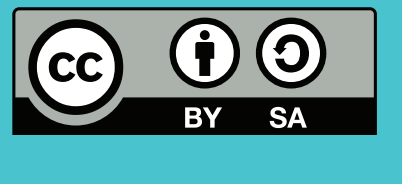

MarXiv is an ОСТO Initiative

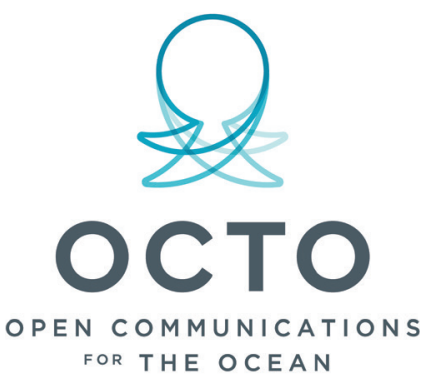

